\title{
BEST POSSIBLE TRIANGLE INEQUALITIES FOR STATISTICAL METRIC SPACES ${ }^{1}$
}

\author{
EDWARD THORP
}

1. A statistical metric space ${ }^{2}$ (briefly, an $S M$ space) is a set $S$ and a mapping $\mathcal{F}$ from $S \times S$ into the set of distribution functions (i.e., real-valued functions of a real variable which are everywhere defined, nondecreasing, left-continuous and have inf 0 and sup 1). The distribution function $\mathscr{F}(p, q)$ associated with a pair of points $(p, q)$ in $S$ is denoted by $F_{p q}$. The functions $F_{p q}$ are assumed to satisfy:

(SM-I) $F_{p q}(x)=1$ for all $x>0$ iff $p=q$.

$(\mathrm{SM}-\mathrm{II}) F_{p q}(0)=0$.

(SM-III) $F_{p q}=F_{q p}$.

(SM-IV) If $F_{p q}(x)=1$ and $F_{q r}(y)=1$, then $F_{p r}(x+y)=1$.

A real-valued function $T$, whose domain is the set of real number pairs $(x, y)$ such that $0 \leqq x, y \leqq 1$, is called a $t$-function if it satisfies the following conditions:

(T-I) $T(a, 1)=a, T(0,0)=0$.

(T-II) $T(c, d) \geqq T(a, b)$ if $c \geqq a, d \geqq b$.

(T-III) $T(a, b)=T(b, a)$ (commutativity).

(T-IV) $T[T(a, b), c]=T[a, T(b, c)]$ (associativity).

Definition 1. A Menger space $(S, \mathfrak{F}, T)$ is an $\operatorname{SM}$ space $(S, \mathfrak{F})$ and a $t$-function $T$ such that the triangle inequality,

$(\mathrm{SM}-\mathrm{IVm}) F_{p r}(x+y) \geqq T\left(F_{p q}(x), F_{q r}(y)\right)$, holds for all points $p, q, r$ in $S$ and for all numbers $x, y \geqq 0$.

Definition 2. The $t$-function $T_{1}$ is stronger than the $t$-function $T_{2}$, and we write $T_{1} \geqq T_{2}$, if $T_{1}(x, y) \geqq T_{2}(x, y)$ for $0 \leqq x, y \leqq 1 ; T_{1}$ is strictly stronger than $T_{2}$ if $T_{1}$ is stronger than $T_{2}$ and there is at least one pair of numbers $(x, y)$ such that $T_{1}(x, y)>T_{2}(x, y)$. Correspondingly, $T_{2}$ is weaker or strictly weaker than $T_{1}$.

For a given SM space there is in general more than one $t$-function which makes it a Menger space. In particular, if $(S, \mathcal{F}, T)$ is a Menger space and $U$ is weaker than $T$, then $(S, \mathcal{F}, U)$ is also a Menger space

Presented to the Society, October 31, 1959; received by the editors October 29, 1959.

1 This research was supported in part by the United States Air Force under contract No. AF-49(638)-42, monitored by the Air Force Office of Scientific Research of the Air Research and Development Command.

2 This concept was introduced by K. Menger in [1] and further developed by B. Schweizer and A. Sklar in [2] and [3]. The author wishes to thank Professors Schweizer and Sklar for helpful conversations and useful suggestions. 
(so that the property of being a Menger space is hereditary under the relation "weaker than").

A $t$-function gives, in a sense, information about the interrelationships between the various distribution functions of the underlying SM space. It follows, in general, that the stronger the Menger function, the more information one has about the structure of the SM space. Hence it is natural to ask whether, given an SM space that can be made into a Menger space, there is a strongest such $t$-function. Similarly it is of interest to know whether, for a given $t$-function there exists a corresponding space for which it is strongest.

In this paper we completely solve the latter problem referred to above by constructing, for a given $t$-function, a space such that it is strongest. The space constructed has an uncountable number of points. However for some $t$-functions it is possible to construct spaces with a finite number of points. We illustrate this by an example, and introduce an alternate construction (not always possible) which is of some interest in itself. The construction for the general case is effected by associating a constraining triangle with each point in the domain of $T$. The alternate construction uses a single triangle to constrain $T$ along a curve or even throughout a region.

The problem of finding a best possible $t$-function for a given SM space is considerably more difficult since it usually depends on the particular form of the distance distribution functions $F_{p q}$. However, a number of results of a general nature can be obtained. These are presented in the second part of this paper, where we also give an example of a Menger space for which there is no strongest $t$-function.

2. TheOREm 1. Let $T$ be a $t$-function. Then there exists a Menger space for which $T$ is the strongest $t$-function satisfying (SM-IVm).

Proof. We construct an SM space such that for every point $(a, b)$ in the unit square, there are points $p, q, r$ in $S$ and numbers $x, y$, such that $F_{p q}(x)=a, F_{q r}(y)=b$, and $F_{p r}(x+y)=T\left[F_{p q}(x), F_{q r}(y)\right]$.

For a fixed $(a, b)$ in the interior of the unit square, choose three points $\{1,2,3\}$. The distribution functions relating these three points are defined as follows:

$$
\begin{aligned}
& F_{12}(x)=\left\{\begin{array}{l}
0, x \leqq 1-\epsilon, \\
a, 1-\epsilon-x \leqq 1, \quad F_{23}(x)=\left\{\begin{array}{l}
0, x \leqq 1-\epsilon, \\
b, 1-\epsilon<x \leqq 1, \\
1,1<x,
\end{array},\right. \\
F_{13}(x)=\left\{\begin{array}{ll}
0, x \leqq 2-2 \epsilon, \\
T(a, b), 2-2 \epsilon<x \leqq 2-\epsilon, \\
1,2-\epsilon<x,
\end{array} \text { where } 0<\epsilon \leqq 1 / 2 .\right.
\end{array}\right.
\end{aligned}
$$


To verify (SM-IVm) for this set of three points, we need only check permutations of the one distinct triple [3, Lemma 2.4]. Moreover, in view of the symmetry of the $F_{p q}$, there are only three distinct permutations. Our task is further simplified because (SM-IVm) is automatically satisfied for those values of $x$ or $y$ making either distribution function on the right zero (since $T(0, a)=0$ ) and for those values of $x+y$ making the distribution function on the left one (since $T(a, b) \leqq 1)$. We check the remaining possibilities.

Case 1. $F_{13}(x+y) \geqq T\left(F_{12}(x), F_{23}(y)\right)$.

$$
1-\epsilon<x \leqq 1 \text { and } 1-\epsilon<y \leqq 1 .
$$

The left side is at least $T(a, b)$ and the right side is at most $T(a, b)$. For what follows it is important to note that equality is attained, for instance, when $x=y=1-\epsilon / 2$.

$$
1-\epsilon<x \leqq 1, \quad 1<y, \text { or } 1-\epsilon<y \leqq 1,1<x .
$$

Then $2-\epsilon<x+y$, so that the left side is 1 .

Case 2. $F_{12}(x+y) \geqq T\left(F_{13}(x), F_{23}(y)\right)$. The right side is 0 unless $x>2-2 \epsilon$ and $y>1-\epsilon$, in which case the left side is 1 .

Case 3. $F_{23}(x+y) \geqq T\left(F_{13}(x), F_{12}(y)\right)$. This is similar to the preceding case.

Next, with every point $(a, b)$ in the interior of the unit square, we associate a triple of points in $S$ whose distance distribution functions are defined as above. Hence each $(a, b)$ has a "triangle" that corresponds to it. We define the distribution function of any pair of points belonging to different triangles to be $H(x-1)$, where $H$ is the distribution function defined by,

$$
H(x)= \begin{cases}0, & x \leqq 0 \\ 1, & x>0 .\end{cases}
$$

The totality of triangles of points so obtained will be the SM space of the theorem. It remains to verify (SM-IVm) for distinct triples involving points from two or three triangles.

If the three triangles are distinct, (SM-IVm) becomes $H(x+y-1)$ $\geqq T(H(x-1), H(y-1))$. The right side is 0 unless $x$ and $y$ both exceed 1 . But then $x+y>1$, so that the left side is 1 .

If there are two triangles, then one of them will contain exactly one point of the triple, say $p$. If $p$ appears on the left in (SM-IVm), then $x+y>1$ makes the left side 1 and $x+y \leqq 1$ makes the right 0 . (Note that this is where we need $\epsilon \leqq 1 / 2$.)

If $p$ does not appear on the left side of (SM-IVm), then it appears in both distribution functions on the right. Hence the right side is 0 
unless both $x$ and $y$ are greater than 1 ; but then $x+y>2$ and the left side is 1 .

We have thus established that our collection of triangles is a Menger space under the given $T$. Furthermore, for this Menger space the given $T$ is the strongest possible. It cannot be strengthened at any point $(a, b)$ in the interior of the unit square, for, by construction, the corresponding triangle is such that for some numbers $x, y$, (SM-IVm) holds with equality when the argument of $T$ is $(a, b)$. This completes the proof of Theorem 1.

It should be noted that some of the properties of $t$-functions, in particular the associativity, were not used in the proof of the theorem. Thus the theorem is true for some functions that are not $t$-functions.

In constraining $T$ so that it cannot be strengthened, the construction in Theorem 1 used one triangle for each point in the domain of $T$. The number of points in the resulting Menger space is thus uncountable. However, for continuous $T$, a space with a countable number of points will do, since it suffices to constrain $T$ on a countable dense subset of the unit square.

It is not known, in general, which continuous functions can be determined by a space with only a finite number of points. That there are continuous functions that can be so constrained is demonstrated by Example 1, below:

EXAMPLE 1. Let $T(a, b)=\max (a+b-1,0) . S=\{1,2,3\}$.

$$
F_{12}(x)=\left\{\begin{array}{ll}
x, & 0<x \leqq 1 \\
1, & 1<x
\end{array}, \quad F_{12}=F_{23} .\right.
$$

According to (SM-IVm), $F_{13}(x+y) \geqq T\left(F_{12}(x), F_{23}(y)\right)$. This suggests that we attempt to constrain $T$ by defining $F_{13}$ to be the smallest function such that the above inequality is true. Thus, let $F_{13}(c)$ $=\max T\left(F_{12}(x), F_{23}(y)\right)$, where $x+y=c$ and $0 \leqq x, y \leqq 1$; then $F_{13}(c)$ $=\max (c-1,0)$ if $c \leqq 2$. Hence $F_{13}$ should be defined by

$$
F_{18}(x)=\left\{\begin{array}{cl}
0, & x<1, \\
x-1, & 1<x \leqq 2, \\
1, & 2<x .
\end{array}\right.
$$

With this definition of $F_{13}$, equality can be obtained in (SM-IVm) for any $x, y$ such that $0<x, y<1 . T(x, y)=T\left(F_{12}(x), F_{23}(y)\right)$ $=\max (x+y-1,0)=F_{13}(x+y)$. Hence $T$ cannot be strengthened at any point. Here a single triple of points constrains $T$ on a twodimensional region (in this case the whole square).

To verify that these definitions of $F_{12}, F_{13}$ and $F_{23}$ are consistent 
with (SM-IVm) for all possible triples of points, only the one triple of distinct points needs to be checked [3, Lemma 2.4]. With $F_{13}$ on the left, (SM-IVm) holds by the definition of $F_{13}$. Since $F_{23}=F_{12}$, it suffices to check $F_{12}(x+y) \geqq T\left(F_{13}(x), F_{23}(y)\right)$ for $x+y<1$. This reduces to $x+y \geqq \max (x+y-1,0)$.

3. Next we investigate the converse of Theorem 1-that is, the question of whether a given Menger space has a strongest $T$ such that $(\mathrm{SM}-\mathrm{IVm})$ holds.

Theorem 2. If $(S, \mathcal{F})$ is a given $S M$ space and $\left\{T_{\alpha}\right\}, \alpha$ in $A$, is a collection of functions, each satisfying (T-I), (T-II), (T-III), and $(\mathrm{SM}-\mathrm{IVm})$ on the space $(S, \mathcal{F})$, then so does sup $T_{\alpha}$.

Proof. The conditions (T-I) and (T-II) imply that the collection $\left\{T_{\alpha}\right\}$ is bounded. The rest now follows since sup $T_{\alpha}$ inherits each of the properties in question.

CoRollary. For every Menger space there is a unique strongest $T$ satisfying all the conditions required of a $t$-function except possibly the associativity condition (T-IV).

EXAMPLE 2. Let $T_{1}(x, y)=\operatorname{Max}(x+y-1,0)$. Let $T_{2}(x, y)=3 / 4$ if $3 / 4 \leqq x, y<1, T_{2}(a, 1)=T_{2}(1, a)=a$, and $T_{2}(x, y)=0$ otherwise. Both $T_{1}$ and $T_{2}$ are readily shown to be associative. However, $T=\max \left(T_{1}, T_{2}\right)$ is not associative because $T[T(3 / 4,3 / 4), 1 / 2]=T(3 / 4,1 / 2)=1 / 4$ while $T[3 / 4, T(3 / 4,1 / 2)]=T(3 / 4,1 / 4)=0$.

This example shows that the sup of two associative functions need not be associative and leads at once to:

Theorem 3. There is a Menger space for which there is no strongest t-function.

Proof. By Theorem 2, the $T$ in Example 2 has all the properties of a $t$-function except associativity. Hence as was noted immediately following the proof of Theorem 1, the construction of that theorem applies to yield an SM space such that this $T$ is the strongest function satisfying (T-I), (T-II), (T-III) and (SM-IVm). This SM space is a Menger space under $T_{1}$ and $T_{2}$. Thus if there were a strongest $t$ function it would be stronger than $T$. Since this is impossible, we have completed the proof.

At present it is not known whether the set of allowable $t$-functions for a Menger space necessarily has a maximal element. We obtain a partial result in this direction (Theorem 4).

Definition 3. A two-place function $F$ is left-continuous if 
$\lim _{x \rightarrow a-} F(x, b)=F(a, b)$ and $\lim _{y \rightarrow b-} F(a, y)=F(a, b)$ for all $(a, b)$ in the domain of $F$.

Theorem 4. If $(S, \mathcal{F})$ is an $S M$ space and $\Im$ the set of all left-continuous $t$-functions $T$ for which $(S, \mathcal{F}, T)$ is a Menger space, then $\mathfrak{J}$ has a maximal element.

Proof. The set $\mathfrak{I}$ is partially ordered by the relation "stronger than" (Definition 2). Thus, by Zorn's Lemma, it suffices to show that the sup of any totally ordered subset of $J$ is again in $J$. Let $\left\{T_{\alpha}\right\}, \alpha$ in $A$, be such a totally ordered subset. The order on $\left\{T_{\alpha}\right\}$ induces an order ( $\leqq$ ) on $A$ via: $\alpha \leqq \beta$ if and only if $T_{\alpha} \leqq T_{\beta}$.

Let $T=\sup T_{\alpha}$. In view of Theorem 2, we have only to show that $T$ is left-continuous and associative.

(1) To show that $T$ is left-continuous, let $x, y, 0 \leqq x, y \leqq 1$, and $\epsilon>0$ be given. Then there is an $\alpha$ such that

$$
0 \leqq T(x, y)-T_{\alpha}(x, y)<\epsilon / 2,
$$

and, because of the left-continuity and monotonicity of $T_{\alpha}$, there is a $\delta>0$ such that for $0<x-x^{\prime}<\delta$,

$$
0 \leqq T_{\alpha}(x, y)-T_{\alpha}\left(x^{\prime}, y\right)<\epsilon / 2 .
$$

Furthermore, from the definition of $T$,

$$
T_{\alpha}\left(x^{\prime}, y\right)-T\left(x^{\prime}, y\right) \leqq 0 .
$$

Combining these three inequalities yields,

$$
T(x, y)-T\left(x^{\prime}, y\right)<\epsilon \text {, whenever } 0<x-x^{\prime}<\delta,
$$

whence, in view of the monotonicity and symmetry, $T$ is left-continuous.

(2) To show that $T$ is associative, it suffices to show that $T\left[T\left(a_{1}, b_{1}\right), c_{1}\right]$ is invariant under any permutation of the elements $\left(a_{1}, b_{1}, c_{1}\right)$. Let $\left(a_{2}, b_{2}, c_{2}\right)$ be such a permutation, and let $\epsilon>0$ be given. Since $J$ is totally ordered, there is a $\beta$ such that

$$
0 \leqq T\left[T\left(a_{i}, b_{i}\right), c_{i}\right]-T_{\beta}\left[T\left(a_{i}, b_{i}\right), c_{i}\right]<\epsilon / 2, \quad i=1,2 ;
$$

and, from the definition of $T$ and the fact that $T_{\beta}$ is left-continuous, there is an $\alpha \geqq \beta$ such that both $T\left(a_{1}, b_{1}\right)-T_{\alpha}\left(a_{1}, b_{1}\right)$ and $T\left(a_{2}, b_{2}\right)$ $-T_{\alpha}\left(a_{2}, b_{2}\right)$ are so small that

$$
0 \leqq T_{\beta}\left[T\left(a_{i}, b_{i}\right), c_{i}\right]-T_{\beta}\left[T_{\alpha}\left(a_{i}, b_{i}\right), c_{i}\right]<\epsilon / 2, \quad i=1,2 .
$$

Since $\alpha \geqq \beta$, it follows that

$$
T_{\beta}\left[T_{\alpha}\left(a_{i}, b_{i}\right), c_{i}\right]-T_{\alpha}\left[T_{\alpha}\left(a_{i}, b_{i}\right), c_{i}\right] \leqq 0, \quad i=1,2 .
$$


Combining the inequalities, we have (remembering that $T=\sup T_{\alpha}$ ),

$$
0 \leqq T\left[T\left(a_{1}, b_{1}\right), c_{1}\right]-T_{\alpha}\left[T_{\alpha}\left(a_{1}, b_{1}\right), c_{1}\right]<\epsilon,
$$

and

$$
0 \leqq T\left[T\left(a_{2}, b_{2}\right), c_{2}\right]-T_{\alpha}\left[T_{\alpha}\left(a_{2}, b_{2}\right), c_{2}\right]<\epsilon .
$$

But $T_{\alpha}$ is associative and symmetric, so that

$$
T_{\alpha}\left[T_{\alpha}\left(a_{1}, b_{1}\right), c_{1}\right]=T_{\alpha}\left[T_{\alpha}\left(a_{2}, b_{2}\right), c_{2}\right] .
$$

Consequently,

$$
T\left[T\left(a_{1}, b_{1}\right), c_{1}\right]-T\left[T\left(a_{2}, b_{2}\right), c_{2}\right]<\epsilon,
$$

from which the result follows.

\section{REFERENCES}

1. K. Menger, Statistical metrics, Proc. Nat. Acad. Sci. U.S.A. vol. 28 (1942) pp. 535-537.

2. B. Schweizer and A. Sklar, Espaces métriques aléatoires, C. R. Acad. Sci. Paris vol. 247 (1948) pp. 2092-2094.

3. - Statistical metric spaces, Pacific J. Math. vol. 10 (1960).

MassachusetTs Institute of Technology 